\title{
RIEMANN PROBLEM FOR ONE-DIMENSIONAL SYSTEM OF CONSERVATION LAWS OF MASS, MOMENTUM AND ENERGY IN ZERO-PRESSURE GAS DYNAMICS
}

\author{
HONGJUN CHENG
}

Abstract. This paper studies the one-dimensional Riemann problem for the system of conservation laws of mass, momentum and energy in zero-pressure gas dynamics. Using the characteristic analysis method, two kinds of solutions are obtained: vacuum and delta-shock solution. Under suitable generalized Rankine-Hugoniot relation and entropy condition, both existence and uniqueness of delta-shock solutions are established. These analytical results well match the results obtained through numerical simulations.

Mathematics subject classification (2010): 35L65, 35L45.

Keywords and phrases: zero-pressure gas dynamics, delta shock wave, generalized Rankine-Hugoniot relation, entropy condition, vacuum.

\section{REFERENCES}

[1] S. Albeverio, O. S. Rozanova, V. M. Shelkovich, Transport and concentration processes in the multidimensional zero-pressure gas dynamics model with the energy conservation law, preprint: arXiv:1101.5815v1 [math-ph] 30 Jan 2011.

[2] F. Bouchut, On zero pressure gas dynamics, Advances in kinetic theory and computing, Series on Advances in Mathematics for Applied Sciences, World Scientific, Singapore, 22 (1994), 171-190.

[3] G. Q. Chen, H. L. LIU, Formation of delta-shocks and vacuum states in the vanishing pressure limit of solutions to the isentropic Euler equations, SIAM J. Math. Anal., 34 (2003), 925-938.

[4] H. J. Cheng, H. C. YAng, Riemann problem for the relativistic Chaplygin Euler equations, J. Math. Anal. Appl., 381 (2011), 17-26.

[5] H. J. Cheng, H. C. YAng, Delta shock waves in chromatography equations, J. Math. Anal. Appl., 380 (2011), 475-485.

[6] H. J. Cheng, H. C. YAng, Delta shock waves as limits of vanishing viscosity for 2-D steady pressureless isentropic flow, Acta Appl. Math, 113 (2011), 323-348.

[7] V. G. DANilov, V. M. SHelKovich, Dynamics of propagation and interaction of $\delta$-shock waves in conservation laws systems, J. Differ. Equations, 221 (2005), 333-381.

[8] V. G. Danilov, V. M. Shelkovich, Delta-shock wave type solution of hyperbolic systems of conservation laws, Q. Appl. Math., 63, 3 (2005), 401-427.

[9] L. H. Guo, W. C. SHEng, T. Zhang, The two-dimensional Riemann problem for isentropic Chaplygin gas dynamic system, Commun. Pure Appl. Anal., 9 (2010), 431-458.

[10] B. T. HAYES, P. G. LEFLOCH, Measure solutions to a strictly hyperbolic system of conservation laws, Nonlinearity, 9 (1996), 1547-1563.

[11] B. L. KeyfitZ, H. C. KRANZER, Spaces of weighted measures for conservation laws with singular shock solutions, J. Differ. Equations, 118 (1995), 420-451.

[12] A. Kraiko, Discontinuity surfaces in medium without self-pressure, Prikladnaia Matematika i Mekhanika, 43 (1979), 539-549. (In Russian) 
[13] J. Q. LI, T. ZHANG, Generalized Rankine-Hugoniot relations of delta-shocks in solutions of transportation equations, Advance in Nonlinear PDE and Related Areas, World Scientific, Singapore, 1998, 219-232.

[14] H. NeSS YAhU, E. TADMOR, Non-oscillatory central differencing for hyperbolic conservation laws, J. Comput. Phys., 87 (1990), 408-463.

[15] B. Nilsson, O. S. Rozanova, V. M. Shelkovich, Mass, momentum and energy conservation laws in zero-pressure gas dynamics and delta-shocks: II, Appl. Anal., 90 (2011), 831-842.

[16] B. Nilsson, V. M. Shelkovich, Mass, momentum and energy conservation laws in zero-pressure gas dynamics and delta-shocks, Appl. Anal., 89 (2010), 1-13.

[17] E. Y. PANOV, V. M. ShelKovich, $\delta^{\prime}$-shock waves as a new type of solutions to systems of conservation laws, J. Differ. Equations, 228 (2006), 49-86.

[18] V. M. SHELKOVICH, The Riemann problem admitting $\delta, \delta^{\prime}$-shocks, and vacuum states (the vanishing viscosity approach), J. Differ. Equations, 231 (2006), 459-500.

[19] W. C. Sheng, T. Zhang The Riemann problem for transportation equation in gas dynamics, Mem. Amer. Math. Soc., 137 (654), (1999), 1-77.

[20] D. C. TAN, T. ZHANG, Two-dimensional Riemann problem for a hyperbolic system of nonlinear conservation laws(I) Four-J cases, J. Differ. Equations, 111 (1994), 203-254.

[21] D. C. TAN, T. ZhANG, Y. X. ZHenG, Delta shock waves as limits of vanishing viscosity for hyperbolic systems of conservation laws, J. Differ. Equations, 112 (1994), 1-32.

[22] H. C. YANG, Riemann problems for a class of coupled hyperbolic systems of conservation laws, J. Differ. Equations, 159 (1999), 447-484.

[23] Y. X. ZHENG, Systems of conservation laws with incomplete sets of eigenvectors everywhere, Advances in nonlinear partial differential equations and related areas (Beijing, 1997): A Volume Dedicated to Professor Xiaqi Ding's 70th Birthday, World Scientific, River Edge, NJ, 1998, 399-426. 